\title{
Sub-angstrom Spatial Resolution in Secondary-electron Imaging Achieved with an Aberration Corrected Scanning Electron Microscope
}

\author{
H. Inada, ${ }^{*}$ D. Su, ${ }^{* *}$ M. Konno, ${ }^{*}$ K. Nakamura, ${ }^{*}$ R.F. Egerton, ${ }^{* * *}$ J. Wall, ${ }^{* *}$ and $\underline{\text { Y. Zhu }}{ }^{* *}$ \\ *Hitachi High Technologies Corp., Ibaraki Japan \\ **Brookhaven National Laboratory, Upton, NY 11973 USA \\ ***University of Alberta, Edmonton, Canada
}

The development of aberration correction has pushed the spatial resolution of electron microscopes below the one angstrom mark. However, the advancement so far has been limited to the use of transmitted electrons, both in scanning (STEM) and stationary (TEM) mode with an improvement of $20-40 \%$. In contrast, the improvement of the spatial resolution using secondary electrons in SEM has been stagnant, although several attempts have been made in recent years. Since the mechanism for the generation of secondary electrons (SE) is traditionally believed through the decay of collective electronic excitations generated by inelastic scattering of the incident high-energy electrons, thus, even for thin samples, the resolution is limited by delocalization to about $1 \mathrm{~nm}$ scale.

We prove this is not the case. We demonstrate using the newly developed Hitachi HD2700C aberration corrected electron microscope [1], it is possible to achieve one angstrom resolution in SE imaging, which represents a fourfold improvement over the best-reported resolution in any SEM. The accomplishment is attributed to better design of electro-optics (including ultra-high electric and mechanical stabilities) and more efficient detectors (Fig.1) [2,3]. Furthermore, the instrument allows us to probe the same sample area using simultaneously secondary electrons emerging from the surface and the transmitted electrons passing through the bulk (Fig.2). The capability of selective visualization of bulk as well as surface atoms is significant. It opens a door to a wide range of applications, such as observation of dopant atoms in electronic devices and study of the active sites and role of individual atoms and their bonding state during a catalytic chemical reaction, especially when combined with synchronized nanoprobe spectroscopy. Quantitative SE image analyses using the corresponding ADF-STEM image as the reference, including measurement of image intensity as a function of applied bias, sample thickness, crystal tilt and defocus will be presented. Possible scattering mechanism to achieve such astonishing resolution will also be discussed [4].

\section{References}

[1] H. Inada, L. Wu, J. Wall, D. Su, and Y. Zhu, Journal of Electron Microscopy, 58, 111 (2009).

[2] Y. Zhu, H. Inada, K. Nakamura, and J. Wall, "Imaging single atoms using secondary electrons with an aberration-corrected electron microscope", Nature Materials, 8808 (2009).

[3] D.C. Joy, "Second best no more", a commentary article highlights the work reported in [2], Nature Materials, 8776 (2009).

[4] Work supported by the U.S. DOE, Office of Basic Energy Science, under Contracts No. DEAC02-98CH10886. 


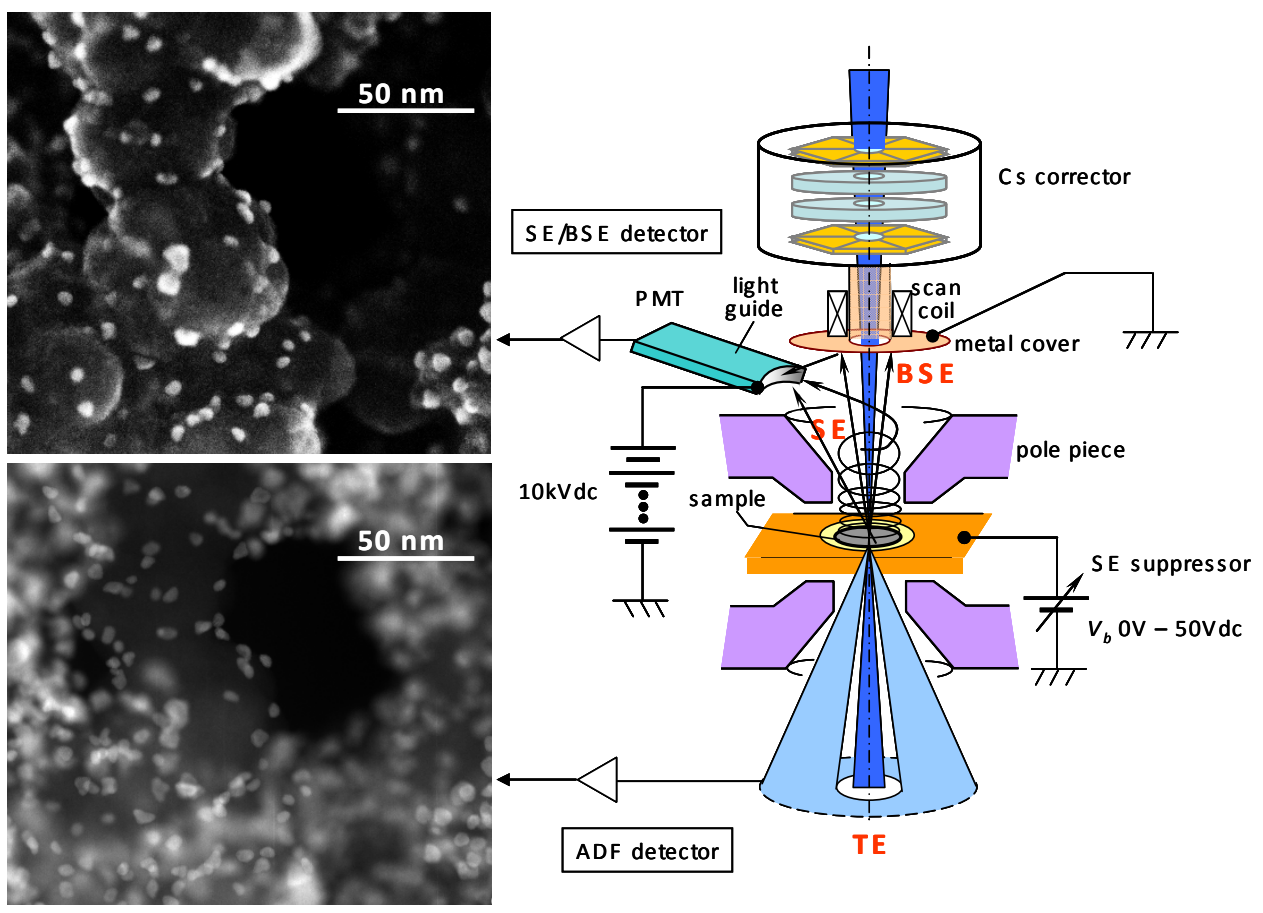

Fig.1 Simultaneous acquisition of SEM image (top left) using secondary electrons (SE), and ADF-STEM image (bottom left) using transmitted electrons (TE). The right panel shows a schematic of the instrument with an in-lens-design pole-piece. The image pair (raw data) shows Pd nanoparticles on a carbon support. We note that the SEM image offers a better three-dimensional view of the area.
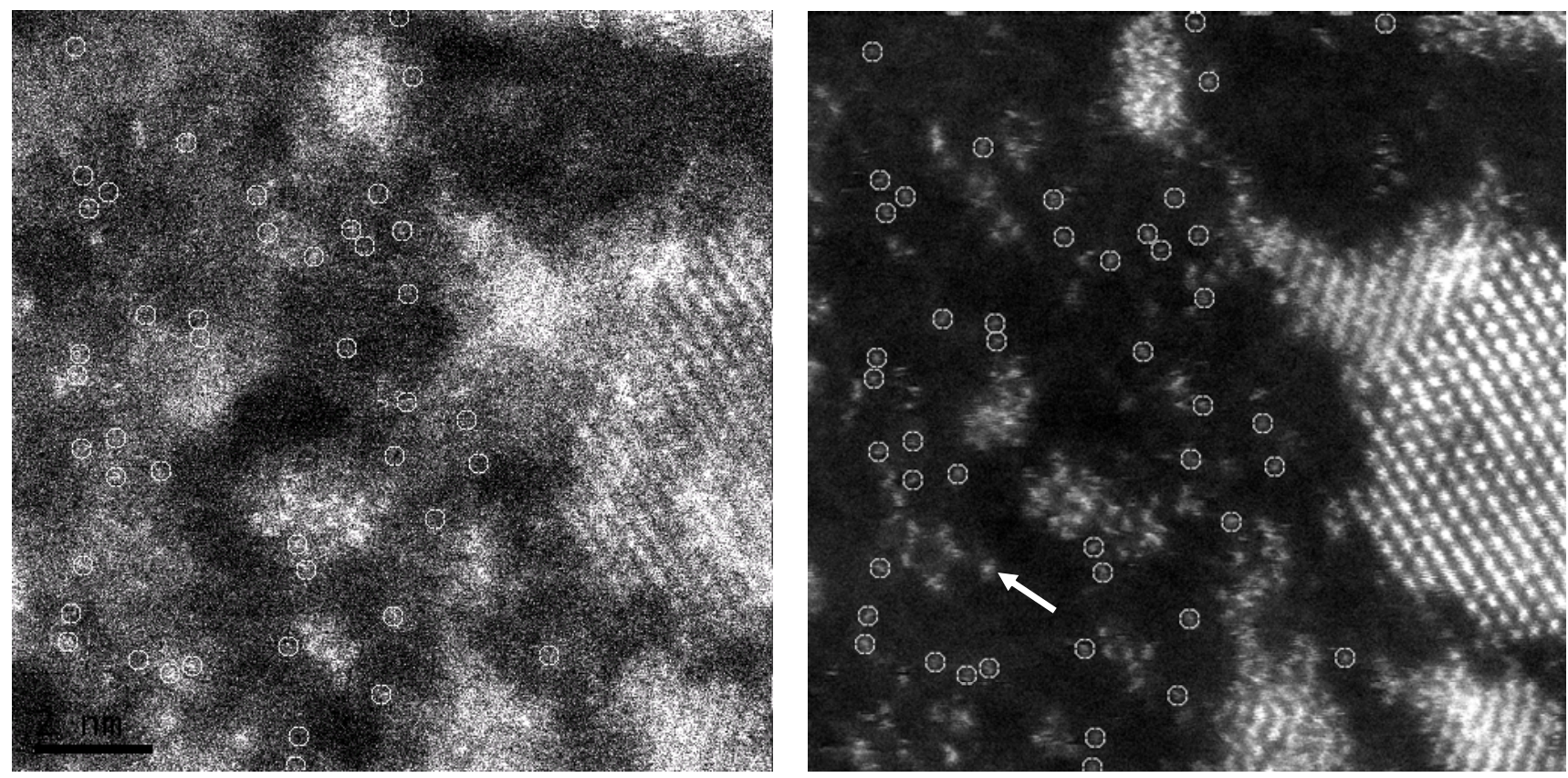

Fig.2 Simultaneous acquisition of the SE image (left) and the ADF-STEM image (right), of uranium oxide nanocrystals and uranium atoms (raw data). Bias experiment reveals that $85-90 \%$ of SE image intensities result from secondary electrons with an energy below $20 \mathrm{eV}$. Quantized intensity analysis in the ADF image suggests the areas marked by small circles are individual isolated uranium atoms. Line profile of average intensity distribution of 354 individual atoms shows FWHM of $0.1 \mathrm{~nm}$ (standard deviation of 20\% with a mean of 14.4). 